\title{
Novel Digital Technologies for Blood Pressure Monitoring and Hypertension Management
}

\author{
Allison J. Hare ${ }^{1,2,3}$ (D) Neel Chokshi ${ }^{1,3,4} \cdot$ Srinath Adusumalli $i^{1,2,3,4}$ \\ Accepted: 28 May 2021 / Published online: 9 June 2021 \\ (C) The Author(s), under exclusive licence to Springer Science+Business Media, LLC, part of Springer Nature 2021
}

\begin{abstract}
Purpose of Review Hypertension is common, impacting an estimated 108 million US adults, and deadly, responsible for the deaths of one in six adults annually. Optimal management includes frequent blood pressure monitoring and antihypertensive medication titration, but in the traditional office-based care delivery model, patients have their blood pressure measured only intermittently and in a way that is subject to misdiagnosis with white coat or masked hypertension. There is a growing opportunity to leverage our expanding repository of digital technology to reimagine hypertension care delivery. This paper reviews existing and emerging digital tools available for hypertension management, as well as behavioral economic insights that could supercharge their impact. Recent Findings Digitally connected blood pressure monitors offer an alternative to office-based blood pressure monitoring. A number of cuffless blood pressure monitors are in development but require further validation before they can be deployed for widespread clinical use. Patient-facing hubs and applications offer a means to transmit blood pressure data to clinicians. Though artificial intelligence could allow for curation of this data, its clinical use for hypertension remains limited to assessing risk factors at this time. Finally, text-based and telemedicine platforms are increasingly being employed to translate hypertension data into clinical outcomes with promising results.

Summary The digital management of hypertension shows potential as an avenue for increasing patient engagement and improving clinical efficiency and outcomes. It is important for clinicians to understand the benefits, limitations, and future directions of digital health to optimize management of hypertension.
\end{abstract}

Keywords Hypertension · Blood pressure - Digital health · Smartphone medical applications · Remote data monitoring · Patient-generated health data

\section{Introduction}

Hypertension is the most common chronic health condition, affecting 108 million (45\%) adults in the USA and 1.4 billion

This article is part of the Topical Collection on Technology and Cardiovascular Health

Allison J. Hare

allison.hare@pennmedicine.upenn.edu

1 Perelman School of Medicine, University of Pennsylvania, Philadelphia, PA, USA

2 Office of the Chief Medical Information Officer, Penn Medicine, Philadelphia, PA, USA

3 Center for Digital Cardiology, Penn Medicine, Philadelphia, PA, USA

4 Division of Cardiovascular Medicine, Department of Medicine, Penn Medicine, Philadelphia, PA, USA
$(31 \%)$ adults globally $[1,2]$. With long-term manifestations including cardiovascular, cerebrovascular, and chronic kidney disease, hypertension has been identified by the World Health Organization as a leading risk factor for morbidity and mortality, responsible for the deaths of one in six adults annually [3]. Despite significant evidence demonstrating the benefits of antihypertensive treatment [4-6], hypertension remains underdiagnosed and undertreated, with an estimated 11 million hypertensive adults unaware of their disease [7] and, of those aware, half surpassing guideline-recommended blood pressure targets $[8,9]$.

Though interventions to improve hypertension screening and management have traditionally been limited to minor adjustments in the current model of office-based care delivery $[10,11]$, alternative models exist. One such model is offered by digital health, defined broadly as "the use of information technology or electronic communication tools, and services and processes to deliver health care services or to facilitate better health" [12]. A number of existing and emerging digital 
health interventions could be applied to any point along the diagnostic and management pathway for hypertension, including mobile applications, text messaging-based interventions, and artificial intelligence-augmented management platforms [13]. Such interventions allow patients to seamlessly measure and share data with their clinicians in a way that is not just different from traditional hypertension screening and management, but potentially better. Home-based blood pressure measurements have been shown to better predict cardiovascular risk, have better reproducibility, and better correlate with measures of end-organ damage compared with officebased measurements [14]. For patients, digital health offers empowerment to participate in their care and reduced rates of misdiagnosis with white coat or masked hypertension. For clinicians, it offers data which may provide a fuller picture of a patient's disease process than what has been historically possible in snapshot clinical settings, as well as the capacity for timelier therapy course corrections before costly care episodes. For health systems, it offers the ability to identify which patients need hypertension interventions from a population health perspective, as well as improved efficiency through a reduction in patient visits for blood pressure measurement or antihypertensive therapy titration alone.

This review article will highlight current applications and efficacy of emerging digital technologies in the detection and management of hypertension, as well as future trends that have potential to shape the nature of clinical practice, increase health system efficiency, and improve patient outcomes.

\section{Emerging Digital Health Solutions for Hypertension Diagnosis and Management}

\section{Patient Identification}

Before hypertensive patients can be treated, they must first be identified. With the passage of the Patient Protection and Affordable Care Act of 2010 as well as the Medicare Access and CHIP Reauthorization Act of 2015 came new payment models that rewarded improved health for populations as a whole rather than the total volume of care provided [15]. This placed a new emphasis on population health management, or the identification of patients with chronic conditions to enable earlier interventions and avoid costly care episodes [16]. Population health management presents a significant challenge to health systems in that many electronic health record (EHR) features were designed to optimize coding and billing rather than quality and outcomes [17], thus making it difficult to identify, stratify, and manage patient populations with chronic diseases such as hypertension. Although the data contained within EHRs hold great potential for applying predictive analytics that could enable real-time determination of the health status of a population, targeted interventions for vulnerable patients, and monitoring of such interventions over time [18], this vision has yet to be realized with the tools at health systems' disposal today in the context of hypertension and beyond.

\section{Data Accrual}

When it comes to diagnosis and management, the most ubiquitous tool in hypertension care is the blood pressure cuff. Traditionally, patients undergo office blood pressure monitoring, where their blood pressure is measured via cuffs in office or kiosk settings. However, a variety of blood pressure cuffs now directly available to patients are equipped with Bluetooth or cellular connectivity, enabling patients to upload their measurements directly to clinicians and bypass the need for officebased monitoring altogether. These cuffs can be used for ambulatory blood pressure monitoring, with measurements taken at set intervals, usually over a period of $24 \mathrm{~h}$, or for home blood pressure monitoring, with measurements taken at a patient's discretion over a longer period.

Home-based blood pressure monitoring holds several advantages over office-based monitoring in the diagnosis of hypertension, which is predicated on multiple longitudinal elevated blood pressure measurements. Appropriate technique for blood pressure measurement is often difficult to implement in the office setting due to a variety of factors, such as rushed visits, and office measurement holds the potential for misdiagnosis with white coat hypertension or masked hypertension. Decades of research have now demonstrated the advantages of home-based over office-based blood pressure monitoring, such as superior predictive ability for hypertension-related morbidity and mortality [19], long-term cardiovascular disease outcomes [20], and left ventricular mass index, a surrogate for left ventricular hypertrophy [21•]. Furthermore, despite evidence that nocturnally elevated blood pressure is a risk factor for adverse cardiovascular events [22], diurnal variation in blood pressure is not captured by one-off office measurements. Thus, more continuous home-based measurements open the window to better understanding patients' variation in blood pressure and recognizing hypertension in those who had not previously carried the diagnosis. Given these factors, clinical guidelines now support the use of home-based readings for confirmations of elevated office blood pressure readings in the diagnosis of hypertension [20]. That said, patients appropriate for home-based blood pressure monitoring should be carefully selected as their ability for self-assessment may be limited by equipment cost, mobility, and health literacy. In such cases, remote monitoring may be less effective compared to traditional models.

Beyond diagnosis, home-based blood pressure monitoring has demonstrated superiority in the management of hypertension. A recent systematic review revealed that blood pressure self-monitoring was associated with a $3.12 \mathrm{mmHg}$ drop in 
systolic blood pressure compared to office-based monitoring at 1 year [23・•]. More qualitative work has demonstrated that blood pressure self-monitoring better enables patients in their care [24] and is considered highly acceptable and usable by patients and their providers [25], increasing patient's diseasespecific knowledge, improving self-management and shared decision-making, and leading to earlier clinical assessment and treatment [26]. However, these benefits should be considered in the context of their potential drawbacks from the patient perspective, including the lost ability for interpersonal contact and the added personal responsibility of remote monitoring platforms [26].

An alternative to cuffed blood pressure monitors is cuffless monitors, which offer a more comfortable and spontaneous mechanism of blood pressure measurement given that their mechanism does not require inflation to occlude arterial pressure. Most cuffless monitors provide continuous calculations of blood pressure using electrocardiography (ECG) signals, photoplethysmogram (PPG) signals, or a combination of both [27]. Because these signals can originate from multiple points on the body, cuffless systems have creatively employed the use of different types of wearables, including sensors that can be positioned behind the ear [28], woven into T-shirts [29], or even placed on one's computer mouse [30]. For example, one system collects PPG signals from a wristband and ECG signals from a belt, then transmits signals via Bluetooth to a mobile application-based algorithm which calculates a continuous estimation of blood pressure [31]. Alternatively, ballistocardiography-based systems entail a user holding a smartphone or watch against their chest wall to estimate the upward recoil of the descending aorta with the downward movement of blood each heartbeat [31], sometimes in conjunction with a patch that captures ECG signals, seismocardiography signals, and body movement via lownoise accelerometers [32]. Other technologies leveraged to cufflessly measure blood pressure include wearable ultrasound patches [33], vascular unloading techniques, tactile sensing, image processing [34], and microfluidic sensorbased systems [35].

\section{Data Transmission}

One step beyond self-monitoring is blood pressure telemonitoring, where patients not only obtain blood pressure measurements in the home setting but also transmit these measurements to their clinicians. The evidence on telemonitoring's added benefit to self-monitoring is mixed. From a qualitative perspective, a large meta-analysis [36] found that telemonitoring had a high degree of acceptance by both clinicians and their patients, as well as high adherence, improved patient outcomes, and reduced healthcare costs. Another meta-analysis demonstrated that telemonitoring in conjunction with co-intervention, such as case management medication titration or counseling, led to a significantly larger and persistent reduction in blood pressure compared to selfmonitoring alone [37]. In 2018, however, the TASMINH4 trial showed statistically insignificant differences in the longterm benefits that self-monitoring and telemonitoring provide. Among patients with poorly controlled hypertension, the use of self-monitoring and management (self-titration of antihypertensive medications), with or without telemonitoring, achieved superior hypertension control compared to titration based on office readings alone. The telemonitoring group achieved lower blood pressures more quickly than the selfmonitoring group, but these were not significantly different at 1 year [38*0]. Additional studies are needed to determine whether the benefits of telemonitoring are sustainable.

Once blood pressure data has been collected for telemonitoring programs, several channels allow patients to transmit this data to their clinicians. Core elements of these channels include patient-facing SMS texting platforms, mobile applications, or Bluetooth/cellular data receivers to which connected blood pressure monitors sync data, as well as servers operated by hosting companies such as Amazon or Microsoft to store data. Texting platforms provide what is arguably the lowest-friction framework for telemonitoring, offering a bidirectional communication channel through which patients can text their providers blood pressure measurements, medication, and symptom data, and providers can text their patients feedback on such data as well as reminders, lifestyle advice, and other forms of support. Such platforms have demonstrated improved efficacy in collecting blood pressure measurements, with one trial demonstrating a 48.5 percentage-point increase in blood pressure measurements obtained for women with hypertensive disorders of pregnancy in the immediate postpartum period compared to women managed with traditional office-based follow-up [39]. Such platforms have also demonstrated efficacy in improving hypertension outcomes [40], with one study reporting a $6.55 \mathrm{mmHg}$ mean drop in systolic blood pressure among patients, no overall increase in workload, a 19\% reduced need for in-person appointments, and a reduced total consultation time by a mean of $15.4 \min [41 \bullet \bullet$.

Hypertension-focused mobile applications offer an alternative mode of blood pressure data tracking and transmission. A recent meta-analysis found that smartphone application-based monitoring interventions led to a significant net improvement in systolic blood pressure and medication adherence [42•], demonstrating the efficacy of this technology. Several applications serve as a window into EHR-based patient portals and allow patients to push blood pressure measurements directly into their EHR record. Many applications additionally offer patient engagement through interactive data visualization and education features, while others are embedded with analytic systems designed to alert clinicians to concerning blood pressure trends. Finally, some applications provide blood pressure 
measurement and medication reminders to patients, which can assist with adherence to antihypertensive regimens. Up to $40 \%$ of patients with chronic medical conditions desire such reminders [43] and many of these applications offer bidirectional functionality, allowing clinicians to monitor their patients' medication adherence and refill needs.

\section{Data Monitoring}

Once patient data has been routed towards a clinician, it requires a carefully designed clinician-facing portal that balances the provision of detailed hypertension data for use in clinical care with the avoidance of overwhelming clinicians with data. To this end, data can be filtered through algorithms that flag problematic trends requiring intervention. When paired with a spectrum of interventions including encouragement, lifestyle tips, or antihypertensive medication titration, this approach to population hypertension management has demonstrated superior efficacy to office-based management: At 90 days, $71 \%$ of digitally managed patients achieved hypertension control compared to $31 \%$ of patients receiving usual care. Notably, patients receiving usual care had an average of 3 blood pressure recordings in the EHR compared with 161 recordings for digitally managed patients. Finally, patient engagement improved with digital management, as demonstrated by a $60 \%$ reduction in patients with low health system activation [44]. Importantly, this precise and continuously interactive approach to population health management is applicable across patient populations regardless of their geographic distribution or historical barriers to care access.

Artificial intelligence (AI) offers a potential mechanism to not only streamline the processing of clinician-facing data, but also draw more powerful insights than what is possible with human attention alone. AI refers to the science of intelligent machine and programming design [45], including natural language processing, machine learning, and deep learning. Recent proliferation in AI use has been driven by increasingly sophisticated deep learning techniques and an exponential increase in available datasets. AI can be applied to vast sets of multimodal data, including genetics, proteomics, behavioral, and environmental factors [46] — all applicable to hypertension given its multifactorial pathogenesis, the complexity of which has impeded preemptive, personalized approaches to the disease's diagnosis and management in the past. Given AI's ability to identify personalized risk factors for hypertension and factors associated with antihypertensive treatment success [46], it has the potential to revolutionize hypertension care. Particularly in the context of monitoring data sent in by patients, AI could filter for concerning trends personalized by patient, allowing for smarter identification of patients in need of intervention, as well as deployment of a more precise intervention itself. Although AI's potential to bring precision medicine to hypertension has been increasingly recognized over the past decade, its use has yet to impact large-scale clinical outcomes [47]. However, as more studies are performed in prospective, large-scale, real-world clinical environments, a future where AI is employed in routine hypertension management is inevitable.

\section{Translating Data to Clinical Outcomes}

Telemedicine, or synchronous virtual interactions between patients and providers via video-based or telephone-based platforms, offers a mechanism for transforming patient-generated blood pressure data into meaningful clinical outcomes. In the context of hypertension care, virtual and in-person visit outcomes have demonstrated similarity - virtually managed hypertensive patients had similar blood pressure control, number of specialist and emergency department visits, and number of inpatient admissions compared to traditionally managed patients [48••]. However, this equivalency presumes that patients are able to transmit blood pressure data to their clinicians. As office visits declined and telemedicine visits increased during the COVID-19 pandemic, there was a $50 \%$ decline in blood pressure assessments and 39\% decline in new hypertension treatment visits [49]. Despite telemedicine visits increasing to compensate for reduced office visits, this shows that management for data-driven diseases like hypertension may have deteriorated, highlighting the need for continued innovation in the multiple mechanisms through which patients can remotely share their health data with their clinicians as described throughout this review.

Though digital health represents a potentially paradigmshifting approach to the diagnosis and management of hypertension, its tools are only as effective as their impact on the behavior of patients and clinicians alike. Given its pathogenesis, hypertension develops and is sustained by suboptimal behaviors. For patients, this can include high salt intake, physical inactivity, or medication non-adherence; for clinicians, this can include inertia in medical decision-making that falls short of best practice guidelines. Therefore, digital health solutions may have a greater impact on hypertension when paired with behavior-influencing insights from behavioral economics, the study of psychology as it relates to individuals' decision-making, and why these decisions are often irrational [50]. Because individuals cannot consistently synthesize complex information to make decisions that maximize their long-term outcomes, they often rely on heuristics which contribute to cognitive biases that lead to predictable decision errors [51]. For example, individuals are motivated more by avoiding losses than receiving equivalent gains [52] and by immediate more than delayed gratification [53], and frequently overestimate the probability of positive events compared to negative ones [54].

Digital health solutions from any point along the hypertension diagnosis and management pathway could combat these 
decision errors, thus optimizing their impact, by incorporating behavioral economic techniques into their design (Fig. 1). For example, hypertensive patient identification tools could increase engagement in population health programs by making patient enrollment opt-out rather than opt-in for clinicians, as individuals are often influenced by a "status quo" bias which nudges them towards the perceived safety of a default option [55]. Commitment contracts can aid individuals in bridging the divide between their current behaviors and future goals [56], so encouraging patients to sign a pre-commitment pledge stating they will do their best to take their blood pressure at home could increase rates of adherence to telemonitoring programs. "Active choice" nudges require individuals to make decisions in real time rather than delaying decisions until later; if patient data is not automatically transmitted to clinicianfacing portals, active choice nudges could be leveraged to prompt patients decide on transmitting their data in real time, thus increasing rates of data submission. In the design of population data monitoring platforms, the principle of salience could be employed to make concerning data trends more visually engaging, thus drawing the clinician's attention to where it is most needed. Finally, translating data into clinical outcomes could be facilitated by "gamification," a behavioral economics strategy that employs game components, such as points, in nongame contexts to motivate individuals to better align short-term with longer-term goals [57]. One group showed that a gamified hypertension intervention was effective in engaging patients to participate in a blood pressure telemonitoring program [58], while another demonstrated a statistically significant relationship between level of achievements earned in a gamified hypertension management platform and decreased blood pressure [59]. Regardless of the point on the hypertension care pathway, those looking to employ digital health solutions would likely optimize their impact by thoughtfully considering principles from behavioral economics.

\section{Implementation Barriers for Digital Health Solutions}

Despite evidence supporting the wide-ranging benefits of digital health solutions for hypertensive patients, adoption has been low on a broad scale. This slow uptake is likely multifactorial, but perhaps the most commonly cited reason is a lack of clear validation standards for devices that claim to capture clinically accurate blood pressure levels. Cuffless blood pressure monitors provide an excellent example of this. Though they provide nearly continuous blood pressure estimations, a lack of rigorous testing of and validation standards for these systems presents barriers to widespread clinical uptake [60]. One such device was recently shown to provide grossly inaccurate blood pressure estimations [61]. Though perhaps simplistic on the surface, blood pressure is a complex metric influenced by numerous factors, which illuminates the pitfall of relying on cuffless measurement methods based on algorithms which factor in a minimal number of physiologic parameters. As these algorithms become more sophisticated, there may come a time when cuffless techniques are usable in a clinical context, but further research and development are necessary before this vision can be realized. Blood pressure mobile applications have also encountered issues when it comes to clinical validation. Though patients can now select from nearly 200 hypertension-focused applications, many are underdeveloped [62]. One review found 108/186 applications examined had unidimensional functionality and nearly all were developed without clinician input [63], while another review found only 3/107 applications examined were developed by healthcare agencies such as academic institutions or professional organizations [64]. This review also found that $15 / 107$ applications claimed an ability to "transform" the smartphone into a medical device and measure blood pressure in a cuffless manner. Of note, no smartphone-based blood pressure measurement methods have been robustly evaluated

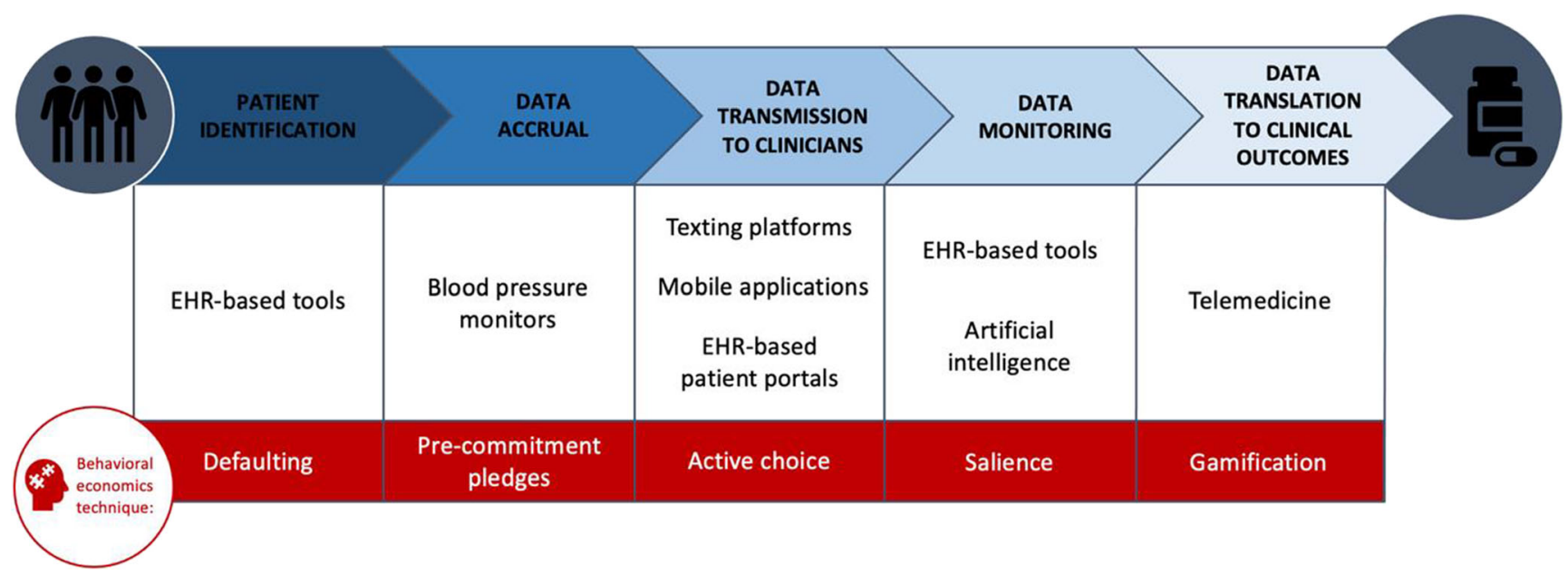

Fig. 1 Central illustration: pathway of patient-generated hypertension data towards a clinician, embedded with examples of behavioral economics techniques that could enhance each step along the pathway 
or obtained approval for use by the US Food and Drug Administration [65]. The American Heart Association has stated that blood pressure measurement applications are unreliable, recording inaccurate measurements four out of five times when one popular application was tested [65]. More work is required before patients can be ensured that all devices claiming to measure their blood pressure are clinically valid, and before clinicians can take blood pressure measurements recorded by unvalidated monitors at face value.

One potential antidote to the issue of validation is the US Blood Pressure Validated Device Listing (VDL) initiative managed by the American Medical Association and the National Opinion Research Center at the University of Chicago. Through an independent review committee of physician experts, blood pressure measurement devices with active FDA 510(k) pre-market clearance are assessed for performance against a set list of "VDL Criteria". Devices that meet criteria are then deemed clinically accurate and compiled into a formal list of devices hosted on the initiative's public-facing site (https://www.validatebp.org/). This represents not only a framework for validating remote blood pressure monitors in the future, but also a resource for clinicians hoping to harness the benefits of remote blood pressure monitoring in their practice at present.

Other barriers to widespread uptake of digital hypertension solutions include lack of robust integration into clinical workflows. Although many solutions offer sleek visual displays of patient data or communication portals, the limited time and cognitive resources of clinicians present a prohibitive barrier to interfaces that require a third-party login or a separate window outside of the EHR. This pitfall is illustrated by a failed trial that intended to investigate the effectiveness of a mobile application for patients with diabetes and hypertension, where low enrollment and inconsistent use of the application by patients barred the trial from completion. The authors found that there was insufficient time during routine care for clinical staff to familiarize patients with the application or check the data it hosted, meaning many patients did not use the application appropriately and, even when they did, data went unused. The application was not integrated into the EHR, which presented an issue for both patients and staff, who said that the application was "just one more thing to attend to" [66]. This trial underscores the need for an understanding of the contextual factors and workflow integrations that impact the ultimate utilization of digital health technologies, as well as the need for usability testing prior to the deployment of similar interventions.

The interoperability enabled by APIs like SMART on FHIR presents a potential solution for integration issues; however, lack of coordination with EHR vendors and financial barriers often prevent this solution from coming to fruition. Despite these challenges, efforts are ongoing to streamline digital and clinical workflows. The EMPOWER trial, for example, is investigating an automated remote monitoring intervention for patients with heart failure using electronic pill bottles to measure diuretic adherence and electronic scales to measure daily weights [67]. Data from these devices flow directly into the health system's EHR through a data gating system, where abnormal values are routed to clinician's inboxes and normal values are routed to the result section of a patient's chart, thus mirroring existing clinical workflows. Patients can be engaged to more actively participate in the management of their disease as well. For example, Allen et al. sent heart failure patients "activation tools" encouraging them to "make one positive change" in their prescriptions before a cardiology visit had a significantly higher rate of initiation or intensification of guideline-directed medical therapies compared to patients in usual care $(49.0 \%$ vs. $29.7 \%, P$ $=0.001$ ) [68]. These represent some of numerous technical frameworks in development that could easily be scaled to the remote monitoring of hypertensive patients, and thus prevent workflow integration issues from being a prohibitive barrier to the full employment of these technologies in the future.

Another important implementation barrier is the prohibitive cost of some devices, which compounds existing inequities that have long existed in the US healthcare system [69]. VDL-approved blood pressure monitors with Bluetooth or cellular data connectivity, for example, cost anywhere between $\$ 50$ and $\$ 200$, and represent only a fraction of the equipment necessary to stand up a comprehensive homemonitoring program. In this same vein, the historical lack of a comprehensive reimbursement policy for hypertension telemonitoring represented a second layer of the financial barriers to the widespread uptake of digital hypertension management. In the post-COVID-19 landscape, however, the Centers for Medicare and Medicaid Services and other payers have implemented billing codes for telemedical care, remote patient monitoring, and even specifically for home blood pressure telemonitoring [70], thus relieving this barrier. Though it is too soon to say whether these recently instituted fee codes will become a permanent part of US healthcare reimbursement policy, the pandemic has certainly opened the door to clinician's ability to envision what could be made possible in clinical practice if virtual care solutions have parity to in-person care models in the future.

\section{Conclusion and Summary}

Given the increasing prevalence, high morbidity and mortality, and underdiagnosed and undertreated nature of hypertension, innovative solutions are needed to optimize the traditional office-based care model that has likely contributed to these suboptimal outcomes. Digital health offers an effective mechanism for more scalable, holistic, and efficient hypertension care delivery, outperforming office-based care models for 
blood pressure control and patient engagement. Here, we described digital health strategies for patient identification, data accrual and transmission to clinicians, data monitoring interfaces, and platforms that help translate data into clinical outcomes for hypertensive patients, while also recognizing the benefits that principles from behavioral economics could add to these technologies. Uptake of these technologies will likely increase considerably in the coming years given the practicality they have demonstrated during the COVID-19 pandemic, benefits they hold over traditional models of care, and increasing technological literacy of the US population. Despite this, much work remains in validating these technologies for widespread use, eliminating cost barriers for patients, and minimizing reimbursement and workflow integration barriers for clinicians.

\section{Declarations}

Conflict of Interest Allison Hare and Drs. Neel Chokshi and Sri Adusumalli declare that they have no conflict of interest.

Human and Animal Rights and Informed Consent This article does not contain any studies with human or animal subjects performed by the authors.

\section{References}

Papers of particular interest, published recently, have been highlighted as:

- Of importance

•. Of major importance

1. Centers for Disease Control and Prevention (CDC). Hypertension cascade: hypertension prevalence, treatment and control estimates among US adults aged 18 years and older applying the criteria from the American College of Cardiology and American Heart Association's 2017 Hypertension Guideline-NHANES 20132016. Atlanta: US Department of Health and Human Services. p. 2019.

2. Mills KT, Bundy JD, Kelly TN, Reed JE, Kearney PM, Reynolds $\mathrm{K}$, et al. Global disparities of hypertension prevalence and control: a systematic analysis of population-based studies from 90 countries. Circ Res. 2016;134:441-50.

3. Organisation WH. World Health Organization (2013), A global brief on hypertension. Report. 2013 April 2013. Contract No.: WHO/DCO/WHD/2013.2.

4. GBD 2015 Risk Factors Collaborators. Global, regional, and national comparative risk assessment of 79 behavioural, environmental and occupational, and metabolic risks or clusters of risks, 19902015: a systematic analysis for the Global Burden of Disease Study 2015. Lancet. 2016;388:1659-724.

5. Law MR, Morris JK, Wald NJ. Use of blood pressure lowering drugs in the prevention of cardiovascular disease: meta-analysis of 147 randomised trials in the context of expectations from prospective epidemiological studies. BMJ. 2009;338:b1665.
6. Collins R, Peto R, MacMahon S, Godwin J, Qizilbash N, Collins R, et al. Blood pressure, stroke, and coronary heart disease. Part 2, short-term reductions in blood pressure: overview of randomised drug trials in their epidemiological context. Lancet. 1990;335:82738 .

7. Ciemins EL, Ritchey MD, Joshi VV, Loustalot F, Hannan J, Cuddeback JK. Application of a tool to identify undiagnosed hypertension-United States. Morb Mortal Wkly Rep. 2018;67: 798-802.

8. Kung $\mathrm{HC}, \mathrm{Xu}$ J. Hypertension-related mortality in the United States, 2000-2013. NCHS Data Brief. 2015;193:1-8.

9. Wolf-Maier K, Cooper RS, Kramer H, Banegas J́R, Giampaoli S, Joffres MR, et al. Hypertension treatment and control in five European countries, Canada and the United States. Hypertension. 2004:43:10-7.

10. Pearson TA, Palaniappan LP, Artinian NT, et al. American Heart Association Guide for Improving Cardiovascular Health at the Community Level 2013 update: a scientific statement for public health practitioners, healthcare providers, and health policy makers. Circ Res. 2013;127:1730-53.

11. A population-based policy and systems change approach to prevent and control hypertension. Washington, DC: Institute of Medicine; 2010.

12. Canada Health Infoway (2020). What is digital health? https:// www.infoway-inforoute.ca/en/what-we-do/benefits-of-digitalhealth/what-is-digital-health. Accessed 28 Jan 2021.

13. Bhavnani SP, Narula J, Sengupta PP. Mobile technology and the digitization of healthcare. Eur Heart J. 2016;37:1428-38.

14. Pickering TG, Miller NH, Ogedegbe G, Krakoff LR, Artinian NT, Goff D, et al. Call to action on use and reimbursement for home blood pressure monitoring: executive summary: a joint scientific statement from the American Heart Association, American Society Of Hypertension, and Preventive Cardiovascular Nurses Association. Hypertension. 2008;52:1-9.

15. Hibbard JH, Greene J, Sacks RM, Overton V, Parrotta C. Improving population health management strategies: Identifying patients who are more likely to be users of avoidable costly care and those more likely to develop a new chronic disease. Health Serv Res. 2017;52:1297-309.

16. Kindig D, Stoddart G. What is population health? Am J Public Health. 2003;93:380-3.

17. Fields RW, Gandhi N. Tools for population health management. Prim Care. 2019;46:529-38.

18. Parikh RB, Kakad M, Bates DW. Integrating predictive analytics into high-value care: the dawn of precision delivery. JAMA. 2016;315:651-2.

19. Muntner P, Shimbo D, Carey RM, et al. Measurement of blood pressure in humans: a scientific statement from the American Heart Association Hypertension. Hypertension. 2019;73:e35-66.

20. Whelton PK, Carey RM, Aronow WS, et al. ACC/AHA/AAPA/ $\mathrm{ABC} / \mathrm{ACPM} / \mathrm{AGS} / \mathrm{APhA} / \mathrm{ASH} / \mathrm{ASPC} / \mathrm{NMA} / \mathrm{PCNA}$ Guideline for the prevention, detection, evaluation, and management of high blood pressure in adults: a report of the American College of Cardiology/American Heart Association Task Force on Clinical Practice Guidelines. Hypertension. 2017;2018(71):e13-e115.

21. Schwartz JE, Muntner P, Kronish IM, et al. Reliability of office, home, and ambulatory blood pressure measurements and correlation with left ventricular mass. J Am Coll Cardiol. 2020;76:2911-22 Schwartz et al. demonstrate that systolic and diastolic blood pressures recorded at home were more reliable and more strongly associated with left ventricular mass index, a surrogate for left ventricular hypertrophy, than blood pressures recorded in the office or 24-h ambulatory settings. The authors suggest that 1 week of home blood pressure monitoring is therefore the optimal approach to diagnosing hypertension. 
22. Boggia J, Li Y, Thijs L, Hansen TW, Kikuya M, BjörklundBodegård K, et al. Staessen JA; International Database on Ambulatory blood pressure monitoring in relation to Cardiovascular Outcomes (IDACO) investigators. Prognostic accuracy of day versus night ambulatory blood pressure: a cohort study. Lancet. 2007;370(9594):1219-29.

23.• Sheppard JP, Tucker KL, Davison WJ, et al. Self-monitoring of blood pressure in patients with hypertension-related multi-morbidity: systematic review and individual patient data meta-analysis. Am J Hypertens. 2020;33:243-51 Sheppard et al. conducted a systematic review of 22 trials and found that blood pressure self-monitoring was associated with a mean $3.12 \mathrm{mmHg}$ drop in blood pressure among patients with hypertension-related comorbidities.

24. McManus RJ, Mant J, Haque MS, Bray EP, Bryan S, Greenfield $\mathrm{SM}$, et al. Effect of self-monitoring and medication self-titration on systolic blood pressure in hypertensive patients at high risk of cardiovascular disease. JAMA. 2014;312:799-808.

25. Albrecht L, Wood PW, Fradette M, McAlister FA, Rabi D, Boulanger P, et al. Usability and acceptability of a home blood pressure telemonitoring device among community-dwelling senior citizens with hypertension: qualitative study. JMIR Aging. 2018;1: e10975.

26. Walker RC, Tong A, Howard K, Palmer SC. Patient expectations and experiences of remote monitoring for chronic diseases: systematic review and thematic synthesis of qualitative studies. Int J Med Inform. 2019;124:78-85.

27. Stojanova A, Koceski S, Koceska N. Continuous blood pressure monitoring as a basis for ambient assisted living (AAL) - review of methodologies and devices. J Med Syst. 2019;43:24.

28. Zhang Q, Zhou D, Zeng X. Highly wearable cuff-less blood pressure and heart rate monitoring with single-arm electrocardiogram and photoplethysmogram signals. Biomed Eng Online. 2017;16:23.

29. Nemati S, Lehman LW, Adams RP, Malhotra A. Discovering shared cardiovascular dynamics within a patient cohort. Conf Proc: Annual International Conference of the IEEE Engineering in Medicine and Biology Society. 2012:6526-9.

30. Kim J, Park J, Kim K, Chee Y, Lim Y, Park K. Development of a nonintrusive blood pressure estimation system for computer users. Telemed J E Health. 2007;13:57-64.

31. Mukkamala R, Hahn JO, Inan OT, Mestha LK, Kim CS, Toreyin H, et al. Toward ubiquitous blood pressure monitoring via pulse transit time: theory and practice. IEEE Trans Biomed Eng. 2015;62:1879901.

32. Etemadi M, Inan OT. Wearable ballistocardiogram and seismocardiogram systems for health and performance. J Appl Physiol. 2018;124:452-61.

33. Wang C, Li X, Hu H, Zhang L, Huang Z, Lin M, et al. Monitoring of the central blood pressure waveform via a conformal ultrasonic device. Nat Biomed Eng. 2018;2:687-95.

34. Mukherjee R, Ghosh S, Gupta B, Chakravarty T. A universal noninvasive continuous blood pressure measurement system for remote healthcare monitoring. Telemed J E Health. 2018;24:803-10.

35. Digiglio P, Li R, Wang W, Pan T. Microflotronic arterial tonometry for continuous wearable non-invasive hemodynamic monitoring. Ann Biomed Eng. 2014;42:2278-88

36. Omboni S, Gazzola T, Carabelli G, Parati G. Clinical usefulness and cost effectiveness of home blood pressure telemonitoring: meta-analysis of randomized controlled studies. J Hypertens. 2013:31:455-67.

37. Tucker KL, Sheppard JP, Stevens R, Bosworth HB, Bove A, Bray EP, et al. Self-monitoring of blood pressure in hypertension: a systematic review and individual patient data meta-analysis. PLoS Med. 2017;14:e1002389.

38.• McManus RJ, Mant J, Franssen M, et al. Efficacy of self-monitored blood pressure, with or without telemonitoring, for titration of antihypertensive medication (TASMINH4): an unmasked randomised controlled trial. Lancet. 2018;391:949-59 This randomized control trial conducted by McManus et al. demonstrated that antihypertensive medication titration guided by blood pressure self-monitoring, with or without the addition of telemonitoring, led to significantly lower blood pressures than antihypertensive medication titration guided by officebased blood pressure monitoring alone.

39. Hirshberg A, Downes K, Srinivas S. Comparing standard officebased follow-up with text-based remote monitoring in the management of postpartum hypertension: a randomised clinical trial. BMJ Qual Saf. 2018;27:871-7.

40. Chow CK, Redfern J, Hillis GS, Thakkar J, Santo K, Hackett ML, et al. Effect of lifestyle-focused text messaging on risk factor modification in patients with coronary heart disease: a randomized clinical trial. JAMA. 2015;314:1255-63.

41.• Hammersley V, Parker R, Paterson M, et al. Telemonitoring at scale for hypertension in primary care: an implementation study. PLoS Med. 2020;17:e1003124 Hammersley et al. demonstrated that blood pressure telemonitoring led to a $6.55 \mathrm{mmHg}$ mean drop in systolic blood pressure among patients with no overall increase in provider workload, a $19 \%$ reduced need for inperson appointments, and a reduced total consultation time by a mean of 15.4 min compared to hypertensive patients managed through routine primary care.

42. Xu H, Long H. The effect of smartphone app-based interventions for patients with hypertension: systematic review and meta-analysis. JMIR Mhealth Uhealth. 2020;19:e21759 Xu et al. conducted a systematic review and meta-analysis on the efficacy of smartphone applications for achieving blood pressure control, medication adherence, and lifestyle changes for hypertensive patients. Their pooled analysis demonstrated a significant overall effect in favor of smartphone application intervention for blood pressure levels and medication adherence, but no overall effect on patients' levels of physical activity.

43. Milani RV, Bober RM, Lavie CJ. The role of technology in chronic disease care. Progr Cardiovasc Dis. 2016;58:579-83.

44. Milani RV, Lavie CJ, Bober RM, Milani AR, Ventura HO. Improving hypertension control and patient engagement using digital tools. Am J Med. 2017;130:14-20.

45. McCarthy J. What is artificial intelligence? Stanford: Stanford University; 2007.

46. Chaikijurajai T, Laffin LJ, Tang WHW. Artificial intelligence and hypertension: recent advances and future outlook. Am J Hypertens. 2020;33:967-74.

47. Matsuoka R, Akazawa H, Kodera S, Komuro I. The dawning of the digital era in the management of hypertension. Hypertens Res. 2020;43:1135-40.

48.•- Levine DM, Dixon RF, Linder JA. Association of structured virtual visits for hypertension follow-up in primary care with blood pressure control and use of clinical services. J Gen Intern Med. 2018;33:1862-7 Levine et al. compared hypertensive patients managed through virtual visits with those managed through usual care, finding that virtually managed patients had fewer primary care office visits but no significant differences in systolic blood pressure control or number of specialist visits, emergency department visits, or inpatient admissions.

49. Alexander GC, Tajanlangit M, Heyward J, Mansour O, Qato DM, Stafford RS. Use and content of primary care office-based vs telemedicine care visits during the COVID-19 pandemic in the US. JAMA Netw Open. 2020;3:e2021476.

50. Loewenstein G, Brennan T, Volpp KG. Asymmetric paternalism to improve health behaviors. JAMA. 2007;298:2415-7.

51. Tversky A, Kahneman D. Judgment under uncertainty: heuristics and biases. Science. 1974;185:1124-31. 
52. Kahneman D, Tversky A. Prospect theory: an analysis of decision under risk. Econometrica. 1979;47:263-92.

53. O'Donoghue T, Rabin M. Doing it now or later. Am Econ Rev. 1999;89:103-24.

54. Sharot $\mathrm{T}$. The optimism bias: a tour of the irrationally positive brain. New York: Pantheon; 2011.

55. Dean M, Kıbris Ö, Masatlioglu Y. Limited attention and status quo bias. J Econ Theory. 2017;169:93-127.

56. Rogers T, Milkman KL, Volpp KG. Commitment devices: using initiatives to change behavior. JAMA. 2014;311:2065-6.

57. Kawachi I. It's all in the game - the uses of gamification to motivate behavior change. JAMA Intern Med. 2017;177:1593-4.

58. Cechetti NP, Bellei EA, Biduski D, Rodriguez JPM, Roman MK, de Marchi ACB. Developing and implementing a gamification method to improve user engagement: a case study with an mHealth application for hypertension monitoring. Telemat Inform. 2019;41:126-38.

59. Siddiqui K, Goglia R. How does patient engagement and gamification correlate with hypertension control? Results from a large-scale nationwide network of ambulatory blood pressure kiosks. Hypertension. 2015;66:AP223.

60. Padwal R. Cuffless blood pressure measurement: how did accuracy become an afterthought. Am J Hypertens. 2019;32:807-9.

61. Federal Trade Commission (2016). Marketers of blood pressure app settle FTC charges regarding accuracy of app readings. https://www.ftc.gov/news-events/press-releases/2016/12/ marketers-blood-pressure-app-settle-ftc-charges-regarding. Accessed 28 Jan 2021.

62. Kitt J, Fox R, Tucker KL, McManus RJ. New approaches in hypertension management: a review of current and developing technologies and their potential impact on hypertension care. Curr Hypertens Rep. 2019;21:44.

63. Alessa T, Hawley MS, Hock ES, de Witte L. Smartphone apps to support self-management of hypertension: review and content analysis. JMIR Mhealth Uhealth. 2019;7:e13645.
64. Kumar N, Khunger M, Gupta A, Garg N. A content analysis of smartphone-based applications for hypertension management. J Am Soc Hypertens. 2015;9:130-6.

65. Jamaladin H, van de Belt TH, Luijpers LC, et al. Mobile apps for blood pressure monitoring: systematic search in app stores and content analysis. JMIR Mhealth UHealth. 2018;6:e187.

66. Thies K, Anderson D, Cramer B. Lack of adoption of a mobile app to support patient self-management of diabetes and hypertension in a federally qualified health center: interview analysis of staff and patients in a failed randomized trial. JMIR Hum Factors. 2017;4: e24.

67. Mehta SJ, Volpp KG, Asch DA, et al. Rationale and design of EMPOWER, a pragmatic randomized trial of automated hovering in patients with congestive heart failure. Circ Cardiovasc Qual. 2019;12:e05126.

68. Allen LA, Venechuk G, Mcllvennan CK, Page RL, Knoepke CE, Helmkamp LJ, et al. An electronically delivered patient-activation tool for intensification of medications for chronic heart failure with reduced ejection fraction: the EPIC-HF trial. Circulation. 2021;143(5):427-37.

69. Abdullah A, Liew SM, Hanafi NS, Ng CJ, Lai PS, Chia YC, et al. What influences patients' acceptance of a blood pressure telemonitoring service in primary care? A qualitative study. Patient Prefer Adherence. 2016;10:99-106.

70. Shimbo D, Artinian NT, Basile JN, et al. Self-measured blood pressure monitoring at home: A joint policy statement from the American Heart Association and American Medical Association. Circ Res. 2020;142:e42-63.

Publisher's Note Springer Nature remains neutral with regard to jurisdictional claims in published maps and institutional affiliations. 